\title{
(1) \\ Upper Normal Limits of Serum Alanine Aminotransferase in Healthy Population: A Systematic Review
}

\author{
Shadi Kolahdoozan ${ }^{1}$, Babak Mirminachi ${ }^{1}$, Sadaf G. Sepanlou ${ }^{1}$, Reza Malekzadeh ${ }^{1}$, Shahin Merat ${ }^{1}$, \\ Hossein Poustchi ${ }^{1, *}$ \\ 1. Digestive Diseases Research Center, \\ Digestive Diseases Research Institute, \\ Tehran University of Medical Sciences, \\ Tehran, Iran

\section{ABSTRACT}

\section{BACKGROUND} \\ Measuring serum alanine aminotransferase (ALT) enzyme is a routine clinical test commonly \\ used to evaluate abnormalities in the body in general, and in the liver function in particular. Higher \\ ALT levels are associated with some metabolic disorders. The upper limit normal (ULN) is considered \\ as a reliable threshold for the definition of high ALT.
}

* Corresponding Author:

Hossein Poustchi, MD

Shariati Hospital, North Kargar Ave.,

14117-13135 Tehran, Iran

Telefax: +982182415104

Fax: + 982182415400

Email: masoumi7415@gmail.com. massoumij@sums.ac.ir

Received: 27 Dec. 2019

Accepted: 10 May. 2020
OBJECTIVES:

To assess the existing evidence on the ULN for ALT in the general population.

DATA SOURCE:

PubMed (Medline), EMBASE, Scopus, and Web of Science (ISI) were searched using a specified search strategy.

\section{ELIGIBILITY CRITERIA:}

We collected documents published from 1980 to 2018 in the English language, focusing on human samples at the population level and extracted the data after qualitative evaluation.

\section{METHODS}

We conducted this study in accordance with the recommendations of the Preferred Reporting Items for Systematic Reviews and Meta-analysis (PRISMA) statement. We used specific search terms and their combinations to find documents from relevant databases. We used a snowballing approach to find documents not captured in the main phase of the search. Two authors separately conducted the search, screened the articles, and selected documents that were qualified for data extraction based on the defined inclusion criteria. Finally, data extraction was conducted by two authors using PRISMA checklist. Reported ULNs for ALT and 95\% confidence intervals (CIs) were documented in previously developed datasheets.

\section{RESULTS}

Out of 15242 studies, 47 articles were included for data extraction and analysis. Data were sparse and lacked the consistency to precisely estimate ULN for serum ALT. The ULN of ALT was significantly diverse across various geographical locations and sexes. The lowest value of ULN for ALT was $19 \mathrm{IU} / \mathrm{L}$ in Chinese children (age range: 7 to $<10$ years), and the highest value of ULN for ALT was $55 \mathrm{IU} / \mathrm{L}$ in children from Ghana aged $<5$ years.

\section{LIMITATIONS:}

The main limitation of the current systematic review was the scarcity of the reported measures for ULN of ALT.

\section{CONCLUSION}

Based on the results of the current systematic review, it is suggested that the normal range of ALT be redefined, but this redefinition should be done according to the localized data. In order to redefine the ULN for ALT, regional differences, methods used in ALT measurements, and ULN determination should be considered.

\section{KEYWORDS:}

Alanine Transaminase, Alanine Aminotransferase, SGPT, ALT, Liver Enzymes

Please cite this paper as:

Kolahdoozan S, Mirminachi B, G. Sepanlou S, Malekzadeh R, Poustchi H, Merat S. Upper Normal Limits of Serum Alanine Aminotransferase in Healthy Population: A Systematic Review. Middle East J Dig Dis 2020;12:194-205. doi: 10.34172/mejdd.2020.182. 


\section{INTRODUCTION}

Measuring liver aminotransferase enzymes levels is an integral part of the screening and diagnosis of liver disease. Liver injury, in any form and duration, finally results in an increase in the plasma level of liver aminotransferases. The aspartate aminotransferase (AST) is found in the liver, cardiac and skeletal muscles, kidneys, brain, and pancreas, in descending order. The highest level of alanine aminotransferase (ALT) enzyme is found in the liver, although non-hepatic causes of ALT elevation may also occur during myopathies. ${ }^{1}$

ALT enzyme is commonly used to diagnose liver injuries and evaluate overall health. Among different liver-related enzymes, ALT is probably the most specific predictor of liver damage, due to its exclusive production site, which is the liver. Therefore, it is necessary to define a clear-cut uniform upper normal range for ALT. Liver cell injuries are responsible for most, but not all the cases of increased ALT levels. ${ }^{2-5}$ Studies show a possible association between ALT and the components of metabolic syndrome, $\mathrm{i}$ e, fasting blood glucose, and serum lipoproteins. People with persistently elevated ALT are at higher risk of metabolic syndrome. ${ }^{6}$ Elevated ALT is assumed as a predictor of metabolic syndrome or type 2 diabetes. ${ }^{2,4,5}$ In addition, previous evidence showed a positive relationship between ALT and overall mortality. Strong populationbased studies suggest ALT as a measure of overall survival and mortality risk. ${ }^{7,8}$

There are controversies regarding the upper limit of normal (ULN) definition and measurement of ALT across insurance companies, health-care providers, and internists, and thus, there is no unique consensus on the ULN of ALT. A 2-fold variation is reported in different reference values..$^{9,10}$

The ULN for ALT was estimated to be $40 \mathrm{IU} / \mathrm{mL}$ based on older population-based studies in which liver biopsy was not performed, and liver inflammation due to viral hepatitis infection and fatty liver disease were not considered. ${ }^{8,11}$ Further studies suggested lower healthy ALT levels. ${ }^{12}$ Clinical studies suggested that the current thresholds for ALT were not adequately effective to distinguish liver abnormalities and to evaluate disease progression in patients with chronic liver conditions such as fatty liver disease. ${ }^{13}$ Several studies investigated the distribution of ALT in different populations to define reference values and variations at individual level. ${ }^{12}$ The exact level of ALT to be considered clinically normal is still doubted, and lab techniques could also affect the reported level of ALT. ${ }^{14}$

In this systematic review, we aimed at gathering data from studies that investigated the reference ranges of ALT in human samples and updating the definition of ULN for ALT based on these studies.

\section{MATERIALS AND METHODS}

\section{Protocol}

Prospective, retrospective cohorts studies with populationbased controls, and cross-sectional population-based studies were evaluated. Reviews and animal studies were excluded. In order to collect all eligible documents that might have been missed by the search strategy, the reference lists in the retrieved articles, reviews, and textbooks were searched and evaluated in this systematic review.

\section{Eligibility criteria}

All population-based studies assessing ALT level in the general adult population through any diagnostic blood tests were examined in this review. Adult general population refers to individuals older than 18 years old without reference to any specific characteristic and with the same male to female ratio.

\section{Information sources}

PubMed (Medline), EMBASE, Scopus, and Web of Science databases were searched using the search strategy considering Cochrane and PubMed subject filtering guidelines. ${ }^{15,16}$ In addition to the above-mentioned databases, the grey literature; i e, thesis databases, and Google Scholar were also searched.

\section{Study selection}

The search results from all databases were combined in an EndNote file, and duplicates were excluded. Two separate reviewers evaluated the documents to be included based on their titles/abstracts. The kappa statistics was used to calculate the degree of agreement between the two reviewers (kappa $=67 \%$ ). The quality of included studies was assessed by two review authors 
using a modified STROBE checklist. Any disagreement was resolved by consensus.

\section{Data extraction}

Data extraction forms were designed according to the PRISMA guideline 17 to extract the following data from the selected articles: study design, publication date, sample size, demographic and anthropometric characteristics of participants, mean values or ULN for ALT, and 95\% confidence intervals (CIs) (if available).

\section{RESULTS}

Following the defined search strategy, 15282 articles were collected for review. After removing duplicates, by screening titles and abstracts, out of 123 remaining documents, 50 studies were selected for full-text appraisal. After quality assessment, 47 qualified articles reporting either mean or ULN for ALT level, were evaluated at the final step (Table 1). ${ }^{7,12-14,18-58}$ This process was summarized in a flowchart (Figure 1).

Overall, 14 studies reported mean ALT and 41 reported ULN (Table 1). The study participants were selected from the general population only in four studies. , $^{7,25,33,44}$ The study participants were among healthy blood donors or liver donors in 15 studies. 13, 19-21, 23, 24, 31, 32, 34, 35, 37-39, 46, 59

The lowest ULN in the overall population, males and females, was 19 in children (age range: 7 to $<10$ years from China, ${ }^{59}$ followed by 17.1 in the subjects aged 9-18 years from Sweden, ${ }^{43}$ and 17.0 in people with the mean age of $52 \pm 13.1$ years from Taiwan. ${ }^{49}$ Moreover, the highest ULN was 55.0 in a population aged $<5$ years from Ghana, ${ }^{60}$ followed by 65.0 in children $<1$ year old from Pakistan in both male and female participants. ${ }^{30,49}$

Measures of dispersion were not adequately reported in selected studies; therefore, a meta-analysis could not be performed.

\section{DISCUSSION}

Overall, the current systematic review found 47 articles reporting either upper normal limit or mean ALT level. Almost in all studies, female participants had lower ULN than male participants. Although there were diversities in the reported ALT levels, in most studies, the ULNs were lower than the current standard limits both in male and female participants. Most studies suggested some adjustments to the current thresholds of ALT to provide a more effective tool to screen liver cell injuries, particularly non-alcoholic fatty liver disease (NAFLD).

The ALT activity depends on demographic variables such as sex, age, height, weight (mainly in men), and the use of specific medications. ${ }^{8,30,49}$ The variations in the reported ULNs might not be totally due to regional differences. In other words, since some of the studies selected their participants from specific groups such as hospital inpatients or blood/liver donors, their results cannot be generalized to the total population. As mentioned by Neuschwander-Tetri and colleagues, ${ }^{10}$ utilization of blood samples obtained from frequent blood donors to calculate ALT normal thresholds might result in misleading thresholds.

Some studies excluded participants with particular characteristics; i e, people with very high or very low body mass index (BMI) or the ones with any kind of chronic conditions. Prati and others, ${ }^{13}$ in a study on blood-bank records recommended that people with an elevated BMI, waist-to-hip ratio, or any components of metabolic syndrome should not be included in the reference population when defining the healthy ALT range.

The need for redefining ULN for ALT originates from the need to reassess the sensitivity and specificity of liver enzymes in screening patients with hepatitis $\mathrm{B}$ virus (HBV) or hepatitis C virus (HCV) infections, or NAFLD. The current "normal" range of ALT may underestimate the presence of these common causes of liver inflammation and chronic liver disease. ${ }^{61}$

Different definitions of "normal" persons to be included in the study population are one of the key parts that cause variations in ULN for ALT in different studies. For example, in a large population-based retrospective study by Kariv and co-workers, ${ }^{12}$ the objective was to show the "healthy" ULN for ALT in Israel. The study information was gathered from medical records of about 272.000 participants. Three groups were defined. Group 1 consisted of the total population of 272.273 subjects. Group 2, which included 87.020 subjects, comprised the total population after exclusion of subjects with abnormal values in at least one laboratory parameter, medical diagnoses that might affect liver function tests, or a medication profile consisting of potentially hepatotoxic drugs. Subjects with abnormal levels of serum triglycerides, cholesterol, glucose, or 
Table 1: Characteristics of studies that had reported mean ALT (ALT level IU/L)

\begin{tabular}{|c|c|c|c|c|c|c|c|c|c|c|c|c|c|c|c|}
\hline \multirow{2}{*}{ Country } & \multirow{2}{*}{ Author } & \multirow{2}{*}{ Year } & \multirow{2}{*}{$\begin{array}{c}\text { ULN } \\
\text { definition } \\
\text { method }\end{array}$} & \multirow{2}{*}{$\begin{array}{c}\text { Samples } \\
\text { and } \\
\text { inclusion } \\
\text { criteria }\end{array}$} & \multirow{2}{*}{$\begin{array}{c}\text { Analyzer/ } \\
\text { Kit }\end{array}$} & \multicolumn{3}{|c|}{ Samples } & \multirow{2}{*}{$\begin{array}{c}\text { Age } \\
\text { Range/ } \\
\text { Mean }\end{array}$} & \multirow[b]{2}{*}{ overall } & \multicolumn{2}{|l|}{ Mean } & \multicolumn{3}{|c|}{ ULN } \\
\hline & & & & & & overall & Male & Female & & & Male & Female & Overall & Male & Female \\
\hline USA & $\begin{array}{l}\text { Ruhl, } \\
\text { C.E.4+ }\end{array}$ & 2012 & $\begin{array}{l}\text { ROC } \\
\text { curve }\end{array}$ & $\begin{array}{c}\text { General } \\
\text { popula- } \\
\text { tion }\end{array}$ & $\begin{array}{c}\text { Hitachi } \\
\text { model } \\
917 \text { multi- } \\
\text { channel } \\
\text { analyzer }\end{array}$ & 18,518 & - & - & $\begin{array}{c}46.5 \pm \\
6.4\end{array}$ & - & - & - & - & 29.0 & 22.0 \\
\hline \multirow{6}{*}{ Korea } & $\begin{array}{l}\text { Kang, } \\
\text { H.S. } .^{53}\end{array}$ & 2011 & $\begin{array}{c}95^{\mathrm{th}} \\
\text { percentile }\end{array}$ & $\begin{array}{c}\text { Healthy } \\
\text { cohort }\end{array}$ & - & 7,403 & - & - & $\begin{array}{c}48.0 \pm \\
12.3\end{array}$ & - & - & - & - & 31.0 & 23.0 \\
\hline & $\begin{array}{l}\text { Park, } \\
\text { H.N. }{ }^{40}\end{array}$ & 2012 & $\begin{array}{c}95^{\mathrm{th}} \\
\text { percentile }\end{array}$ & $\begin{array}{c}\text { Healthy } \\
\text { subjects } \\
\text { with } \\
\text { normal } \\
\text { weight }\end{array}$ & $\begin{array}{c}\text { ADI- } \\
\text { VIA1650 } \\
\text { analyzer }\end{array}$ & 27,913 & - & - & $20-69$ & - & 34.0 & 25.0 & - & - & - \\
\hline & $\begin{array}{c}\text { Kim, } \mathrm{H} . \\
\text { C.? }\end{array}$ & 2004 & $\begin{array}{l}\text { ROC } \\
\text { curve }\end{array}$ & $\begin{array}{l}\text { Prospec- } \\
\text { tive } \\
\text { cohort } \\
\text { study } \\
\text { (Health } \\
\text { insurance } \\
\text { data) }\end{array}$ & - & 142,055 & - & - & $35-59$ & - & - & - & - & 30.0 & $<30$ \\
\hline & $\begin{array}{l}\text { Sohn, } \\
\text { W. }^{46}\end{array}$ & 2013 & $\begin{array}{c}95^{\mathrm{th}} \\
\text { percentile }\end{array}$ & $\begin{array}{c}\text { Healthy } \\
\text { blood } \\
\text { donors }\end{array}$ & - & 411,240 & - & - & $\begin{array}{c}24.6 \pm \\
6.4\end{array}$ & - & - & - & - & 34.0 & 24.0 \\
\hline & $\begin{array}{l}\text { Park, } \\
\text { S.H. }{ }^{41}\end{array}$ & 2012 & $\begin{array}{c}95^{\mathrm{th}} \\
\text { percentile }\end{array}$ & $\begin{array}{c}\text { Cross- } \\
\text { sectional }\end{array}$ & - & 3,316 & - & - & $10-19$ & - & 53.0 & 30.0 & - & 33.0 & 25.0 \\
\hline & $\begin{array}{l}\text { Lee, } \\
\text { J.K. }{ }^{35}\end{array}$ & 2010 & $\begin{array}{c}97.5^{\text {th }} \\
\text { percentile }\end{array}$ & $\begin{array}{c}\text { Healthy } \\
\text { liver } \\
\text { donors }\end{array}$ & $\begin{array}{c}\text { TBA } \\
\text { 200FR } \\
\text { NEO auto } \\
\text { analyzer } \\
\text { (Toshiba, } \\
\text { Tokyo, } \\
\text { Japan) }\end{array}$ & 1,105 & 643 & 462 & $\begin{array}{c}29.1 \pm \\
9.0\end{array}$ & - & - & - & - & 35.0 & 26.0 \\
\hline \multirow{6}{*}{ Taiwan } & $\begin{array}{c}\text { Wu, } \\
\text { W.C. }{ }^{49}\end{array}$ & 2012 & $\begin{array}{c}95^{\text {th }} \\
\text { percentile }\end{array}$ & $\begin{array}{c}\text { Cross- } \\
\text { sectional } \\
\text { (large- } \\
\text { scale } \\
\text { popula- } \\
\text { tion } \\
\text { based) }\end{array}$ & - & 34,346 & - & - & $52.4 \pm 13.1$ & - & - & - & - & 21.0 & 17.0 \\
\hline & \multirow{5}{*}{$\begin{array}{l}\text { Lai } \\
\text { DS. }\end{array}$} & \multirow{5}{*}{2009} & \multirow{5}{*}{$\begin{array}{c}97.5^{\text {th }} \\
\text { percentile }\end{array}$} & $\begin{array}{l}\text { students } \\
\text { and their } \\
\text { teachers }\end{array}$ & - & 4,326 & 2138 & 2188 & - & - & - & - & 38.0 & 41.0 & 36.0 \\
\hline & & & & Teachers & - & 348 & - & - & $20 \leq$ & - & - & - & 49.1 & - & - \\
\hline & & & & \multirow{3}{*}{$\begin{array}{l}\text { Healthy } \\
\text { students/ } \\
\text { children }\end{array}$} & & 325 & - & - & $13-15$ & - & - & - & 29.7 & - & - \\
\hline & & & & & - & 1,624 & - & - & $9-12$ & - & - & - & 29.6 & - & - \\
\hline & & & & & & 2,029 & - & - & $3-6$ & - & - & - & 24.0 & - & - \\
\hline \multirow{3}{*}{ China } & $\begin{array}{l}\text { Zheng, } \\
\text { M.H. } .^{52}\end{array}$ & 2012 & $\begin{array}{c}95^{\mathrm{h}} \\
\text { percentile }\end{array}$ & $\begin{array}{c}\text { Cross- } \\
\text { sectional }\end{array}$ & $\begin{array}{c}\text { Hitachi } \\
7600 \\
\text { automatic } \\
\text { Analyzer } \\
\text { (Hitachi, } \\
\text { Japan) }\end{array}$ & 53,037 & - & - & $19-44$ & - & 35.0 & 23.0 & - & - & - \\
\hline & $\begin{array}{l}\text { Zhang, } \\
\text { G.M. } .^{50}\end{array}$ & 2014 & $\begin{array}{c}97.5^{\text {th }} \\
\text { percentile }\end{array}$ & $\begin{array}{l}\text { Healthy } \\
\text { popula- } \\
\text { tion }\end{array}$ & - & 54,912 & - & - & $65-104$ & - & - & - & - & 47.3 & 45.2 \\
\hline & $\begin{array}{c}\text { Zhang, } \\
\text { P. }^{51}\end{array}$ & 2015 & $\begin{array}{l}\text { ROC } \\
\text { curve }\end{array}$ & $\begin{array}{l}\text { Non- } \\
\text { random } \\
\text { samples }\end{array}$ & - & 37,69 & - & - & $\begin{array}{c}45.0 \pm \\
19.0\end{array}$ & - & - & - & - & 22.15 & 22.40 \\
\hline UK & $\begin{array}{c}\text { Mijovic } \\
\mathrm{V}^{2}{ }^{2}\end{array}$ & 1987 & $\begin{array}{l}\text { Not } \\
\text { reported }\end{array}$ & $\begin{array}{c}\text { Frequent } \\
\text { blood } \\
\text { donors }\end{array}$ & $\begin{array}{c}\text { Epos } \\
\text { automated } \\
\text { clinical } \\
\text { analyzer } \\
\text { at } 37^{\circ} \mathrm{C}\end{array}$ & 2023 & & & & & & & & & \\
\hline
\end{tabular}




\section{Upper Normal Limits of ALT in Healthy Population}

\begin{tabular}{|c|c|c|c|c|c|c|c|c|c|c|c|c|c|c|c|}
\hline \multirow[b]{2}{*}{ Country } & \multirow[b]{2}{*}{ Author } & \multirow[b]{2}{*}{ Year } & \multirow{2}{*}{$\begin{array}{c}\text { ULN } \\
\text { definition } \\
\text { method }\end{array}$} & \multirow{2}{*}{$\begin{array}{c}\text { Samples } \\
\text { and } \\
\text { inclusion } \\
\text { criteria }\end{array}$} & \multirow{2}{*}{$\begin{array}{c}\text { Analyzer/ } \\
\text { Kit }\end{array}$} & \multicolumn{3}{|c|}{ Samples } & \multirow{2}{*}{$\begin{array}{c}\text { Age } \\
\text { Range/ } \\
\text { Mean }\end{array}$} & \multicolumn{3}{|c|}{ Mean } & \multicolumn{3}{|c|}{ ULN } \\
\hline & & & & & & overall & Male & Female & & $\begin{array}{l}\text { over- } \\
\text { all }\end{array}$ & Male & Female & Overall & Male & Female \\
\hline \multirow{8}{*}{ UK } & \multirow{6}{*}{$\begin{array}{c}\text { Mijovic } \\
\mathrm{V}^{2}{ }^{2}\end{array}$} & \multirow{6}{*}{1987} & \multirow{6}{*}{$\begin{array}{c}\text { Not } \\
\text { reported }\end{array}$} & \multirow{6}{*}{$\begin{array}{c}\text { Frequent } \\
\text { blood } \\
\text { donors }\end{array}$} & \multirow{6}{*}{$\begin{array}{c}\text { Epos } \\
\text { automated } \\
\text { clinical } \\
\text { analyzer } \\
\text { at } 37^{\circ} \mathrm{C}\end{array}$} & \multirow{6}{*}{2023} & 1088 & 935 & $18-65$ & $\begin{array}{c}18.0 \pm \\
12.8\end{array}$ & $\begin{array}{c}22.3 \pm \\
14.7\end{array}$ & $\begin{array}{c}13.4 \pm \\
7.7\end{array}$ & - & - & - \\
\hline & & & & & & & 200 & 247 & $18-25$ & - & $\begin{array}{c}20.0 \pm \\
13.3\end{array}$ & $\begin{array}{c}12.4 \pm \\
6.8\end{array}$ & - & - & - \\
\hline & & & & & & & 292 & 240 & $26-35$ & - & $\begin{array}{c}22.5 \pm \\
9.5\end{array}$ & $\begin{array}{c}13.2 \pm \\
7.1\end{array}$ & - & - & - \\
\hline & & & & & & & 317 & 235 & $36-45$ & - & $\begin{array}{c}24.1 \pm \\
17.2\end{array}$ & $\begin{array}{c}13.7 \pm \\
9.0\end{array}$ & - & - & - \\
\hline & & & & & & & 191 & 126 & $46-55$ & - & $\begin{array}{c}20.6 \pm \\
9.9\end{array}$ & $\begin{array}{c}14.5 \pm \\
8.9\end{array}$ & - & - & - \\
\hline & & & & & & & 88 & 74 & $56-65$ & - & $\begin{array}{c}20.3 \pm \\
9.7\end{array}$ & $\begin{array}{c}14.9 \pm \\
5.9\end{array}$ & - & - & - \\
\hline & & & & $\begin{array}{l}\text { Blood } \\
\text { donors }\end{array}$ & Technicon & - & 209 & 155 & $\begin{array}{l}\text { Mean } \\
=31\end{array}$ & - & 20.1 & 14.1 & - & 65.0 & 32.0 \\
\hline & $\begin{array}{l}\text { Goldie } \\
\text { D.J. }{ }^{3}\end{array}$ & 1990 & $\begin{array}{c}97.5^{\mathrm{m}} \\
\text { percentile }\end{array}$ & $\begin{array}{l}\text { Plasma- } \\
\text { pheresis } \\
\text { donors }\end{array}$ & $\begin{array}{l}\mathrm{SMAC} 1 \\
\text { analyzer } \\
\text { at } 37^{\circ} \mathrm{C}\end{array}$ & - & 374 & 193 & $\begin{array}{l}\text { Mean } \\
=33\end{array}$ & - & 23.4 & 15.2 & - & 62 & 46.0 \\
\hline \multirow{4}{*}{ Italy } & Prati, D. ${ }^{13}$ & 2002 & $\begin{array}{l}95^{\text {th }} \text { per- } \\
\text { centile }\end{array}$ & $\begin{array}{c}\text { Blood } \\
\text { donors } \\
\text { with } \\
\text { low risk } \\
\text { of liver } \\
\text { disease }\end{array}$ & $\begin{array}{c}\text { Olympus } \\
\text { AU510 } \\
\text { Analyzer }\end{array}$ & 6,835 & 3865 & 2970 & $\begin{array}{c}29.8 \pm \\
9.5\end{array}$ & - & $\begin{array}{c}17.6 \\
(17.4- \\
17.9)\end{array}$ & $\begin{array}{c}11.0 \\
(10.7- \\
11.3)\end{array}$ & - & 30.0 & 19.0 \\
\hline & $\begin{array}{l}\text { Vespa- } \\
\text { siani- } \\
\text { Gentiluc- } \\
\text { ci, } U .^{48}\end{array}$ & 2014 & $\begin{array}{c}\text { Not } \\
\text { reported }\end{array}$ & $\begin{array}{l}\text { Large- } \\
\text { scale } \\
\text { study }\end{array}$ & - & 44,232 & - & - & $18 \leq$ & - & 28.0 & 20.0 & - & - & - \\
\hline & \multirow{2}{*}{$\begin{array}{l}\text { Dorizzi, } \\
\text { R. M. }{ }^{4}\end{array}$} & \multirow{2}{*}{1991} & $97.5^{\mathrm{th}}$ & \multirow{2}{*}{$\begin{array}{l}\text { Blood } \\
\text { donors }\end{array}$} & & 314 & 209 & 105 & - & - & 21.4 & 14.7 & - & 42.0 & 28.0 \\
\hline & & & tile & & & 311 & 206 & 105 & - & - & 20.0 & 12.1 & - & 43.0 & 23.0 \\
\hline Morocco & $\begin{array}{l}\text { Laouina, } \\
\text { A. }^{34}\end{array}$ & 2012 & $\begin{array}{c}\text { Not } \\
\text { reported }\end{array}$ & $\begin{array}{l}\text { Healthy } \\
\text { blood } \\
\text { donors }\end{array}$ & $\begin{array}{c}\text { Kinetic } \\
\text { method } \\
\text { using flat- } \\
\text { bottomed } \\
\text { micro- } \\
\text { plate tech- } \\
\text { niques }\end{array}$ & 14,071 & - & - & $18-60$ & - & - & - & - & 64.0 & 52.0 \\
\hline \multirow[b]{2}{*}{ Germany } & $\begin{array}{l}\text { Grunen- } \\
\text { berg, } \\
\text { R. }^{24}\end{array}$ & 1995 & $\begin{array}{c}\text { Not } \\
\text { reported }\end{array}$ & $\begin{array}{l}\text { Healthy } \\
\text { blood } \\
\text { donors }\end{array}$ & - & 5,706 & - & - & Adults & - & - & - & - & 45.0 & 45.0 \\
\hline & $\begin{array}{c}\text { Brink- } \\
\text { mann, } \\
\text { T.19 }\end{array}$ & 2003 & $\begin{array}{l}\text { Linear } \\
\text { regres- } \\
\text { sion } \\
\text { model }\end{array}$ & $\begin{array}{l}\text { Healthy } \\
\text { blood } \\
\text { donors }\end{array}$ & - & 333 & - & - & - & - & - & - & - & 43.9 & 34.4 \\
\hline Spain & $\begin{array}{l}\text { Lozano, } \\
\text { M. }^{37}\end{array}$ & 1998 & $\begin{array}{c}97.5^{\mathrm{th}} \\
\text { percen- } \\
\text { tile }\end{array}$ & $\begin{array}{l}\text { Healthy } \\
\text { blood } \\
\text { donors }\end{array}$ & - & 1,036 & 579 & 457 & $18-65$ & - & - & - & - & 56.0 & 34.0 \\
\hline $\begin{array}{l}\text { Milan, } \\
\text { Beijing, } \\
\text { Bursa, } \\
\text { Nordic } \\
\text { Countries }\end{array}$ & $\begin{array}{l}\text { Ceriotti, } \\
\text { F. }^{14}\end{array}$ & 2010 & $\begin{array}{c}97.5^{\mathrm{th}} \\
\text { percen- } \\
\text { tile }\end{array}$ & $\begin{array}{l}\text { Non- } \\
\text { random } \\
\text { laboratory } \\
\text { samples }\end{array}$ & - & 765 & - & - & $18-85$ & - & - & - & - & 59.0 & 41.0 \\
\hline Finland & $\begin{array}{l}\text { Leino, } \\
\text { L. }^{36}\end{array}$ & 1995 & $\begin{array}{c}\text { Trans- } \\
\text { formed } \\
97.5^{\text {th }} \\
\text { percentile }\end{array}$ & $\begin{array}{l}\text { General } \\
\text { popula- } \\
\text { tion }\end{array}$ & - & 954 & - & - & $27-67$ & - & - & - & - & 50.0 & 38.0 \\
\hline \multirow{3}{*}{ Sweden } & \multirow{3}{*}{$\begin{array}{c}\text { Rodoo } \\
\text { P. }\end{array}$} & \multirow{3}{*}{2013} & \multirow{3}{*}{$\begin{array}{c}97.5^{\text {th }} \\
\text { percen- } \\
\text { tile }\end{array}$} & \multirow{3}{*}{$\begin{array}{c}\text { Healthy } \\
\text { individu- } \\
\text { als }\end{array}$} & & 157 & - & 157 & $8 \leq$ & - & - & - & - & - & 22.9 \\
\hline & & & & & - & 173 & 173 & - & 9-18 & - & - & - & - & 17.1 & - \\
\hline & & & & & & 356 & - & - & - & - & - & - & 30 & - & - \\
\hline
\end{tabular}




\begin{tabular}{|c|c|c|c|c|c|c|c|c|c|c|c|c|c|c|c|}
\hline \multirow{2}{*}{ Country } & \multirow{2}{*}{ Author } & \multirow[b]{2}{*}{ Year } & \multirow{2}{*}{$\begin{array}{c}\text { ULN } \\
\text { definition } \\
\text { method }\end{array}$} & \multirow{2}{*}{$\begin{array}{l}\text { Samples } \\
\text { and } \\
\text { inclusion } \\
\text { criteria }\end{array}$} & \multirow{2}{*}{$\begin{array}{c}\text { Analyzer/ } \\
\text { Kit }\end{array}$} & \multicolumn{3}{|c|}{ Samples } & \multirow{2}{*}{$\begin{array}{c}\text { Age } \\
\text { Range/ } \\
\text { Mean }\end{array}$} & \multicolumn{3}{|c|}{ Mean } & \multicolumn{3}{|c|}{ ULN } \\
\hline & & & & & & overall & Male & Female & & $\begin{array}{c}\text { over- } \\
\text { all }\end{array}$ & Male & Female & Overall & Male & Female \\
\hline Sweden & $\begin{array}{c}\text { Helm- } \\
\text { ersson- } \\
\text { Kar- } \\
\text { lqvist, } \\
\text { J.25 }\end{array}$ & 2016 & $\begin{array}{l}97.5^{\text {th }} \\
\text { percen- } \\
\text { tile }\end{array}$ & $\begin{array}{c}\text { General } \\
\text { population }\end{array}$ & - & 531 & 265 & 266 & 80 & - & - & - & - & 35.9 & 34.12 \\
\hline \multirow{6}{*}{ Denmark } & \multirow{6}{*}{$\begin{array}{l}\text { Hilsted } \\
\text { L. }^{6}\end{array}$} & \multirow{6}{*}{2013} & \multirow{6}{*}{$\begin{array}{c}97.5^{\text {th }} \\
\text { percentile }\end{array}$} & \multirow{6}{*}{$\begin{array}{l}\text { Healthy } \\
\text { individuals }\end{array}$} & \multirow{6}{*}{-} & 84 & 36 & 48 & $5-6$ & - & - & - & 24 & 24.0 & 24.0 \\
\hline & & & & & & 228 & 93 & 135 & $7-8$ & - & - & - & 28 & 28.0 & 35.0 \\
\hline & & & & & & 362 & 158 & 204 & $9-10$ & - & - & - & 35 & 37.0 & 32.0 \\
\hline & & & & & & 391 & 164 & 227 & $11-13$ & - & - & - & 32 & 37.0 & 33.0 \\
\hline & & & & & & 198 & 83 & 106 & $14-16$ & - & - & - & 31 & 31.0 & 32.0 \\
\hline & & & & & & 167 & 62 & 105 & $17-19$ & - & - & - & 43 & 64.0 & 31.0 \\
\hline Palestine & $\begin{array}{l}\text { Kariv, } \\
\text { R.'.12 }\end{array}$ & 2006 & $\begin{array}{l}95^{\text {th }} \text { per- } \\
\text { centile }\end{array}$ & $\begin{array}{l}\text { Lab-based } \\
\text { study }\end{array}$ & - & 17,496 & - & - & $15-90$ & - & 22.7 & 16.7 & - & 44.9 & 31.8 \\
\hline $\begin{array}{l}\text { Saudi } \\
\text { Arabia }\end{array}$ & $\begin{array}{c}\text { Al- } \\
\text { hamoudi, } \\
\text { W! }{ }^{18}\end{array}$ & 2013 & $\begin{array}{l}\text { ROC } \\
\text { curve }\end{array}$ & $\begin{array}{c}\text { Healthy } \\
\text { Liver } \\
\text { donors }\end{array}$ & - & 175 & - & - & $\begin{array}{c}29.9 \pm \\
7.3\end{array}$ & - & 25.4 & 17.7 & - & 33.0 & 22.0 \\
\hline \multirow{4}{*}{ Ghana } & \multirow{4}{*}{$\begin{array}{c}\text { Dosoo } \\
\text { DK? }\end{array}$} & \multirow{4}{*}{2014} & \multirow{4}{*}{$\begin{array}{c}97.5^{\text {th }} \\
\text { percen- } \\
\text { tile }\end{array}$} & \multirow{4}{*}{$\begin{array}{c}\text { General } \\
\text { popula- } \\
\text { tion }\end{array}$} & \multirow{4}{*}{ - } & 491 & - & - & $<5$ & - & - & - & 55.0 & - & - \\
\hline & & & & & & 473 & - & - & $5-12$ & - & - & - & 53.0 & - & - \\
\hline & & & & & & 281 & - & - & $13-17$ & - & - & - & & 61.0 & 48.0 \\
\hline & & & & & & 57 & 31 & 26 & Infants & - & - & - & 24.8 & 22.1 & 27.3 \\
\hline \multirow{5}{*}{ Iran } & $\begin{array}{l}\text { Jamali, } \\
\text { R. }^{28}\end{array}$ & 2008 & $\begin{array}{l}95^{\text {th }} \text { per- } \\
\text { centile }\end{array}$ & 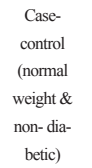 & - & 859 & - & - & $18-75$ & - & - & - & - & 37.5 & 36.0 \\
\hline & $\begin{array}{c}\text { Khedmat, } \\
\mathrm{H.}^{31}\end{array}$ & 2007 & $\begin{array}{c}33.33 \\
\text { and } \\
66.66 \\
\text { Tertiles }\end{array}$ & $\begin{array}{c}\text { Healthy } \\
\text { blood } \\
\text { donors }\end{array}$ & - & 934 & - & - & $18-68$ & - & 33.1 & - & - & 35.0 & - \\
\hline & $\begin{array}{c}\text { Mo- } \\
\text { hamad } \\
\text { nejad, } \\
\text { M. }{ }^{39}\end{array}$ & 2003 & $\begin{array}{l}95^{\text {th }} \text { per- } \\
\text { centile }\end{array}$ & $\begin{array}{c}\text { Healthy } \\
\text { blood } \\
\text { donors, } \\
\text { normal } \\
\text { weight }\end{array}$ & - & 1,939 & - & - & - & - & 21.0 & 16.4 & - & 40.0 & 34.0 \\
\hline & $\begin{array}{l}\text { Kabir, } \\
\text { A. } .^{99}\end{array}$ & 2013 & $\begin{array}{l}95^{\text {th }} \text { per- } \\
\text { centile }\end{array}$ & $\begin{array}{c}\text { Healthy } \\
\text { popula- } \\
\text { tions }\end{array}$ & $\begin{array}{c}\text { Hitachi } \\
704 \text { auto } \\
\text { analyzer, } \\
\text { (To-kyo, } \\
\text { Japan) } \\
\text { with Pars } \\
\text { Azmoon } \\
\text { Reagents } \\
\text { kit } \\
\text { (Tehran, } \\
\text { Iran). }\end{array}$ & 1,309 & - & - & $50<$ & - & - & - & - & 21.4 & 18.8 \\
\hline & $\begin{array}{l}\text { Poust- } \\
\text { chi, } \mathrm{H}^{42}\end{array}$ & 2011 & $\begin{array}{l}95^{\text {h }} \text { per- } \\
\text { centile }\end{array}$ & $\begin{array}{l}\text { Healthy } \\
\text { school } \\
\text { children }\end{array}$ & - & 975 & - & - & $7-18$ & - & - & - & - & 30.0 & 21.0 \\
\hline \multirow{8}{*}{ Pakistan } & \multirow{6}{*}{$\begin{array}{c}\text { Khan, F. } \\
\text { A. }{ }^{8}\end{array}$} & \multirow{6}{*}{1997} & \multirow{6}{*}{$\begin{array}{c}97.5^{\mathrm{th}} \\
\text { percen- } \\
\text { tile }\end{array}$} & & & 90 & - & - & $<1$ & - & - & - & - & 65.0 & 65.0 \\
\hline & & & & & & 370 & - & - & $1-10$ & - & - & - & - & 52.0 & 49.0 \\
\hline & & & & Healthy & & - & - & - & $11-14$ & - & - & - & - & 45.0 & 43.0 \\
\hline & & & & individuals & & 1,452 & - & - & $15-40$ & - & - & - & - & 42.0 & 41.0 \\
\hline & & & & & & - & - & - & $41-60$ & - & - & - & - & 41.0 & 40.0 \\
\hline & & & & & & 203 & - & - & $61-80$ & - & - & - & - & 40.0 & 40.0 \\
\hline & Ibrahim & 1020 & $97.5^{\mathrm{m}}$ per- & Healthy & Hyland & 202 & 114 & 88 & $15 \leq$ & - & - & - & - & 48.0 & 50.0 \\
\hline & K. ${ }^{9}$ & 1988 & centile & individuals & Kit & 137 & 74 & 63 & $<15$ & - & - & - & - & 41.0 & 44.0 \\
\hline
\end{tabular}




\begin{tabular}{|c|c|c|c|c|c|c|c|c|c|c|c|c|c|c|c|}
\hline \multirow{2}{*}{ Country } & \multirow[b]{2}{*}{ Author } & \multirow[b]{2}{*}{ Year } & \multirow{2}{*}{$\begin{array}{c}\text { ULN } \\
\text { definition } \\
\text { method }\end{array}$} & \multirow{2}{*}{$\begin{array}{c}\text { Samples } \\
\text { and } \\
\text { inclusion } \\
\text { criteria }\end{array}$} & \multirow{2}{*}{$\begin{array}{c}\text { Analyzer/ } \\
\text { Kit }\end{array}$} & \multicolumn{3}{|c|}{ Samples } & \multirow{2}{*}{$\begin{array}{c}\text { Age } \\
\text { Range/ } \\
\text { Mean }\end{array}$} & \multirow[b]{2}{*}{ overall } & \multicolumn{2}{|l|}{ Mean } & \multicolumn{3}{|c|}{ ULN } \\
\hline & & & & & & overall & Male & Female & & & Male & Female & Overall & Male & Female \\
\hline \multirow[t]{5}{*}{ India } & $\begin{array}{c}\text { Choud- } \\
\text { hary, } \\
\text { N.S. } .^{20}\end{array}$ & 2014 & $\begin{array}{l}\text { Linear } \\
\text { regres- } \\
\text { sion }\end{array}$ & $\begin{array}{l}\text { Healthy } \\
\text { donors }\end{array}$ & $\begin{array}{c}\text { pyrodoxal }^{5} \\
\text { phosphate } \\
\text { (lactate } \\
\text { dehydroge- } \\
\text { nase/ nico- } \\
\text { tinamide } \\
\text { adenine di- } \\
\text { nucleotide) } \\
\text { reflectance } \\
\text { spectro- } \\
\text { photometry } \\
\text { method } \\
\text { on Vitros } \\
\text { instrument } \\
\text { (Johnson } \\
\text { and } \\
\text { Johnson } \\
\text { USA) }\end{array}$ & 331 & 147 & 184 & $\begin{array}{c}35.7 \pm \\
10.2\end{array}$ & - & - & - & - & 35.0 & 28.0 \\
\hline & $\begin{array}{c}\text { Kumar, } \\
\text { S. }\end{array}$ & 2013 & $\begin{array}{c}\text { Not } \\
\text { reported }\end{array}$ & $\begin{array}{c}\text { Healthy } \\
\text { blood } \\
\text { donors }\end{array}$ & $\begin{array}{c}\text { Olympus } \\
\text { AU400 } \\
\text { auto- } \\
\text { analyzer } \\
\text { (Mishima } \\
\text { Olympus } \\
\text { Co. Ltd., } \\
\text { Shizuoka- } \\
\text { ken, Japan }\end{array}$ & 5,077 & - & - & $18-60$ & - & - & - & - & 30.0 & 19.0 \\
\hline & \multirow{3}{*}{$\begin{array}{c}\text { Mohan } \\
\text { P. }^{10}\end{array}$} & \multirow{3}{*}{2016} & \multirow{3}{*}{$\begin{array}{c}97.5^{\text {th }} \\
\text { percentile }\end{array}$} & \multirow{3}{*}{$\begin{array}{c}\text { Healthy } \\
\text { popula- } \\
\text { tion }\end{array}$} & \multirow{3}{*}{$\begin{array}{c}\text { Sysmex } \\
\text { XN } 3120 \\
7 \text { Part cell } \\
\text { Counter } \\
\text { (Kobe, } \\
\text { Japan) }\end{array}$} & \multirow{3}{*}{2600} & 132 & 104 & $20-35$ & - & - & - & - & $\begin{array}{c}22.23 \pm \\
2.83\end{array}$ & $\begin{array}{c}19.22 \pm \\
3.30\end{array}$ \\
\hline & & & & & & & 30 & 34 & $36-50$ & - & - & - & - & $\begin{array}{c}21.10 \pm \\
2.68\end{array}$ & $\begin{array}{c}19.76 \pm \\
3.22\end{array}$ \\
\hline & & & & & & & 21 & 23 & $>50$ & - & - & - & - & $\begin{array}{c}20.71 \pm \\
3.33\end{array}$ & $\begin{array}{c}19.30 \pm \\
3.66\end{array}$ \\
\hline \multirow[b]{2}{*}{ Australia } & $\begin{array}{c}\text { George, } \\
\text { J.22 }\end{array}$ & 2008 & $\begin{array}{c}95^{\text {h }} \\
\text { percentile }\end{array}$ & $\begin{array}{l}\text { Healthy } \\
\text { students }\end{array}$ & - & 496 & 292 & 204 & $15 \mathrm{y}$ & - & - & - & - & 59.0 & 54.0 \\
\hline & $\begin{array}{c}\text { Van Der } \\
\text { Poorten, } \\
\text { D. }{ }^{47}\end{array}$ & 2007 & $\begin{array}{c}95^{\text {th }} \\
\text { percentile }\end{array}$ & $\begin{array}{l}\text { Healthy } \\
\text { adoles- } \\
\text { cents }\end{array}$ & $\begin{array}{c}\text { Beckman } \\
\text { Synchron } \\
\text { LX } 20\end{array}$ & 209 & - & - & $12-19$ & - & - & - & - & 28.0 & - \\
\hline Eritrea & $\begin{array}{l}\text { Achila, } \\
\text { O.0. }{ }^{55}\end{array}$ & 2017 & & $\begin{array}{l}\text { Healthy } \\
\text { elderly } \\
\text { subjects }\end{array}$ & $\begin{array}{c}\text { Beckman } \\
\text { Coulter: } \\
\text { AU } 480 \\
\text { Chemistry } \\
\text { System }\end{array}$ & 249 & 116 & 133 & & $\begin{array}{l}16.6 \\
(16- \\
17.7)\end{array}$ & $\begin{array}{c}17 \\
(15.8- \\
18.2)\end{array}$ & $\begin{array}{c}16.7 \\
(15.5- \\
18)\end{array}$ & & & \\
\hline Ethiopia & $\begin{array}{c}\text { Abebe, } \\
\text { M. }\end{array}$ & 2018 & & $\begin{array}{c}\text { Healthy } \\
\text { Young, } \\
\text { adult, } \\
\text { blood } \\
\text { donors }\end{array}$ & $\begin{array}{c}\text { Mindray } \\
\text { BS-200E } \\
\text { (Shenzhen } \\
\text { Mindray } \\
\text { Bio- } \\
\text { medical } \\
\text { electronics } \\
\text { co.ltd, } \\
\text { China) }\end{array}$ & 1175 & 644 & 531 & $\begin{array}{c}20(18- \\
22)\end{array}$ & $\begin{array}{c}15.71 \\
(15.22- \\
16.24)\end{array}$ & $\begin{array}{l}17.15 \\
(16.5- \\
17.88)\end{array}$ & $\begin{array}{c}13.97 \\
(13.34- \\
14.74)\end{array}$ & & & \\
\hline \multirow{5}{*}{ China } & \multirow{4}{*}{$\operatorname{LiX}^{*}$} & \multirow{4}{*}{2018} & \multirow{4}{*}{$\begin{array}{c}\text { nonpara- } \\
\text { metric } \\
\text { method }\end{array}$} & \multirow{4}{*}{$\begin{array}{c}\text { healthy } \\
\text { children } \\
\text { aged from } \\
2 \text { to } 14 \\
\text { years }\end{array}$} & \multirow{4}{*}{$\begin{array}{c}\text { Hitachi } \\
7600-210 \\
\text { automatic } \\
\text { biochemi- } \\
\text { cal } \\
\text { analyzer }\end{array}$} & 196 & & & 2 & & & & $\begin{array}{c}24(22- \\
26)\end{array}$ & & \\
\hline & & & & & & 663 & & & $\begin{array}{l}3 \text { to } \\
<6\end{array}$ & & & & $\begin{array}{c}23(21- \\
24)\end{array}$ & & \\
\hline & & & & & & 275 & & & $\begin{array}{c}7 \text { to }< \\
10\end{array}$ & & & & $\begin{array}{c}19(16- \\
22)\end{array}$ & & \\
\hline & & & & & & 113 & & & $\begin{array}{c}11 \text { to }< \\
14\end{array}$ & & & & $\begin{array}{c}25(15- \\
33)\end{array}$ & & \\
\hline & $\mathrm{Li}, \mathrm{Y}^{58}$ & 2018 & $\begin{array}{c}97.5^{\text {th }} \text { per- } \\
\text { centile }\end{array}$ & $\begin{array}{c}\text { Children } \\
\text { aged } \\
12-18 \\
\text { years old } \\
\text { at the } \\
\text { Health } \\
\text { Manage- } \\
\text { ment } \\
\text { Center }\end{array}$ & $\begin{array}{c}\text { Architect } \\
\text { C-8000 } \\
\text { (Abbott } \\
\text { laborato- } \\
\text { ries, USA) } \\
\text { automated } \\
\text { chemistry } \\
\text { analyzer }\end{array}$ & 1682 & & & $12-18$ & & & & 41.5 & 42.8 & 32.8 \\
\hline
\end{tabular}




\begin{tabular}{|c|c|c|c|c|c|c|c|c|c|c|c|c|c|c|c|}
\hline \multirow{2}{*}{ Country } & \multirow{2}{*}{ Author } & \multirow{2}{*}{ Year } & \multirow{2}{*}{$\begin{array}{c}\text { ULN } \\
\text { definition } \\
\text { method }\end{array}$} & \multirow{2}{*}{$\begin{array}{c}\text { Samples } \\
\text { and } \\
\text { inclusion } \\
\text { criteria }\end{array}$} & \multirow{2}{*}{$\begin{array}{l}\text { Analyzer/ } \\
\text { Kit }\end{array}$} & \multicolumn{3}{|c|}{ Samples } & \multicolumn{2}{|l|}{ Age } & \multicolumn{2}{|l|}{ Mean } & \multicolumn{3}{|c|}{ ULN } \\
\hline & & & & & & overall & Male & Female & $\begin{array}{c}\text { Range/ } \\
\text { Mean }\end{array}$ & overall & Male & Female & Overall & Male & Female \\
\hline Germany & $\begin{array}{l}\text { Bussler, } \\
\text { S. }{ }^{56}\end{array}$ & 2018 & $\begin{array}{l}97^{\text {th }} \text { per- } \\
\text { centile }\end{array}$ & $\begin{array}{c}\text { Primarily } \\
\text { healthy } \\
\text { children } \\
\text { and ado- } \\
\text { lescent }\end{array}$ & $\begin{array}{c}\text { cobasR } \\
\text { analyzer } \\
\text { series (pho- } \\
\text { tometric } \\
\text { measur- } \\
\text { ing unit, } \\
\text { c-module, } \\
\text { Roche Di- } \\
\text { agnostics } \\
\text { GmbH, } \\
\text { Mannheim, } \\
\text { Germany). }\end{array}$ & 3131 & 1663 & 1468 & $\begin{array}{c}11 \\
\text { months } \\
\text { to } 16 \\
\text { years }\end{array}$ & & & & & $29.9-38$ & $24.2-31.7$ \\
\hline
\end{tabular}

hemoglobin A1c (HbA1c) were not excluded from this group. Group 3, with 17.496 subjects, comprised only the subjects from group 2 with normal values of triglycerides, cholesterol, glucose, and $\mathrm{HbAlc}$, and thus it included a 'healthy' population. The 95th percentile ALT values in the groups 1, 2, and 3 were respectively $50.1 \mathrm{IU} / \mathrm{L}$ (40.6 for females; 60.8 for males), $40 \mathrm{IU} / \mathrm{L}$ (32.4 for females; 48 for males), and $37.5 \mathrm{IU} / \mathrm{L}$ (31.8 for females; 44.9 for males).

In addition to the known variables such as sex, age, weight, socioeconomic status, and nutritional habits, ${ }^{13,44,62}$ there were other probable confounding factors that would make the results inconsistent. In this regard, Dutta and colleagues mentioned the variety of commercial kits used to evaluate ALT levels as a possible influential factor in the variation of reported ALT normal limits. ${ }^{63}$ Table 1 shows similar diversities in laboratory kits used in different studies enrolled in the current systematic review.

On the other hand, the methodologies used to compute these thresholds were also debatable. Most studies either used the receiver operating characteristic (ROC) curve method or considered $97.5^{\text {th }}$ or $95^{\text {th }}$ percentile to define ULNs (Table 1).

Different studies listed a variety of factors that could modulate the ALT activity and proposed to consider the variables in defining the normal range of ALT. The mentioned variables that may influence the ALT level were age, sex, waist circumference, and BMI. Some authors suggested that factors such as lipid profile; i e, total cholesterol, high-density lipoprotein cholesterol (HDLC), low-density lipoprotein cholesterol (LDL-C), and triglyceride, fasting glucose and insulin level, viral profile; i e hepatitis B surface antigen (HBsAg) and anti-
HCV Ab, alcohol consumption, and the use of potentially hepatotoxic medications should be considered in defining the healthy reference population participating in the calculation of the ULN for ALT. ${ }^{13,47,49}$

Besides the observed differences between reported ULNs for ALT and its current standard limits in most studies, there were variations in the results of studies from the same country, as well. Some evidence peculated the influence of ethnicity on ALT levels in a healthy population, although there were some contradictory discussions regarding this issue. ${ }^{63,64}$ As mentioned before, these diversities might be related to the source cohorts of studies samples. ${ }^{64}$ Due to the growing prevalence of obesity and metabolic syndrome, in which the hepatic component presents as NAFLD and may cause an asymptomatic rise in ALT, it is worthwhile to consider the association between BMI and ALT levels in calculating the normal range for ALT. As an example, among the studies conducted in Iran, one study recruited its participants from a general population with BMI ranging from low to high, while the others restricted their participants only to individuals with normal BMI levels. ${ }^{28,29,31,39,42}$

Many authors suggested that the currently used ULN for ALT does not make an exact clear-cut and true discrimination between the presence and absence of liver disease. Many patients with mild chronic liver disease are not detected using the current ULN for ALT test. More than half of patients with NAFLD, which is now recognized as one of the most common causes of chronic liver disease and cirrhosis in many parts of the world, have serum ALT levels within the current normal limit. Moreover, various population-based studies showed that increased serum ALT levels might be independently 

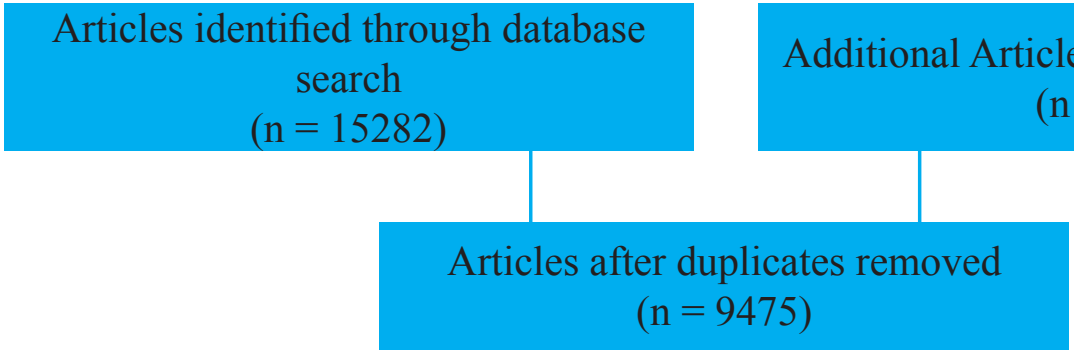
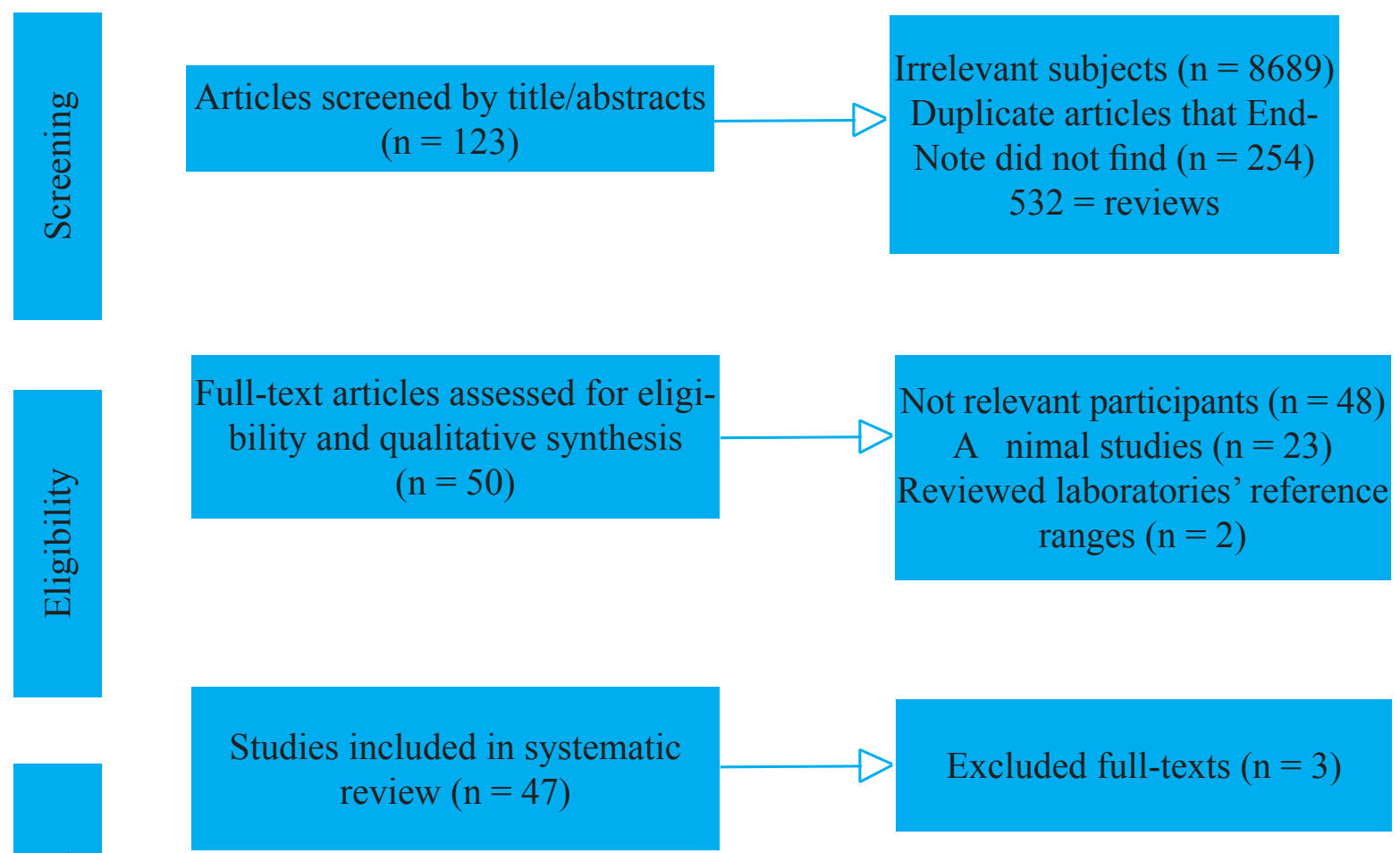

Fig.1: The process of selection eligible studies

related to increased risk of mortality, even within the normal range of ALT level. ${ }^{53,65}$ One recent study showed that people with no liver disease and a slightly elevated, but still normal ALT level, seemed to have an increased all-cause mortality rate. ${ }^{66}$ However, some other studies showed that normal ALT levels were protective. It is important to have a clear definition of normal and abnormal ALT tests to better interpret the results of such studies.

\section{Strengths and limitations}

To the best of the authors knowledge, the current study was the first systematic review in this field.

One limitation of the current review was the number of studies reporting CIs for the estimated ULN in healthy individuals. This withheld authors to perform a metaanalysis to determine a combined weighted value for ULN. Another limitation of the current systematic review was the restriction of this review to studies with healthy samples. This might cause incomplete retrieval of relevant 
studies. Defining criteria for a healthy individual might be different from one study to another.

\section{CONCLUSION}

Since several factors affect ALT level among apparently healthy individuals and even in the tested healthy people, it seems impossible to internationally determine a reliable ULN for serum ALT to be used in all laboratories. There is a need for designing and conducting nationwide studies with identical inclusion criteria to provide reliable and large domestic datasets and estimate a reliable ULN for ALT.

\section{ETHICAL APPROVAL}

There is nothing to be declared.

\section{CONFLICT OF INTEREST}

The authors declare no conflict of interest related to this work.

\section{REFERENCES}

1. Pratt DS, Kaplan MM. Evaluation of abnormal liverenzyme results in asymptomatic patients. $N$ Engl J Med 2000;342:1266-71. doi: 10.1056/NEJM200004273421707.

2. Giannini EG. Liver enzyme alteration: a guide for clinicians. CMAJ 2005;172:367-79. doi: 10.1503/cmaj.1040752.

3. Gowda S, Desai PB, Hull VV, Math AAK, Vernekar SN, Kulkarni SS. A review on laboratory liver function tests. Pan Afr Med J 2009;3:17.

4. Liu Z, Que S, Xu J, Peng T. Alanine aminotransferaseold biomarker and new concept: a review. Int J Med Sci 2014;11:925-35. doi: 10.7150/ijms.8951.

5. Tan KK, Bang SL, Vijayan A, Chiu MT. Hepatic enzymes have a role in the diagnosis of hepatic injury after blunt abdominal trauma. Injury 2009;40:978-83. doi: 10.1016/j. injury.2009.02.023.

6. Hanley AJ, Williams K, Festa A, Wagenknecht LE, D'Agostino RB, Jr., Haffner SM. Liver markers and development of the metabolic syndrome: the insulin resistance atherosclerosis study. Diabetes 2005;54:31407. doi: 10.2337/diabetes.54.11.3140.

7. Kim HC, Nam CM, Jee SH, Han KH, Oh DK, Suh I. Normal serum aminotransferase concentration and risk of mortality from liver diseases: prospective cohort study. BMJ 2004;328:983. doi: 10.1136/bmj.38050.593634.63.

8. Siest G, Schiele F, Galteau MM, Panek E, Steinmetz J, Fagnani F, et al. Aspartate aminotransferase and alanine aminotransferase activities in plasma: statistical distributions, individual variations, and reference values. Clin Chem 1975;21:1077-87.
9. Dutta A, Saha C, Johnson CS, Chalasani N. Variability in the upper limit of normal for serum alanine aminotransferase levels: a statewide study. Hepatology 2009;50:1957-62. doi: 10.1002/hep.23200.

10. Neuschwander-Tetri BA, Unalp A, Creer MH, Nonalcoholic Steatohepatitis Clinical Research N. Influence of local reference populations on upper limits of normal for serum alanine aminotransferase levels. Arch Intern Med 2008;168:663-6. doi:10.1001/archinternmed.2007.131.

11. Sherman KE. Alanine aminotransferase in clinical practice. A review. Arch Intern Med 1991;151:260-5. doi:10.1001/ archinte.1991.00400020036008.

12. Kariv R, Leshno M, Beth-Or A, Strul H, Blendis L, Kokia E, et al. Re-evaluation of serum alanine aminotransferase upper normal limit and its modulating factors in a largescale population study. Liver Int 2006;26:445-50. doi: 10.1111/j.1478-3231.2006.01197.x

13. Prati D, Taioli E, Zanella A, Della Torre E, Butelli S, Del Vecchio E, et al. Updated definitions of healthy ranges for serum alanine aminotransferase levels. Ann Intern Med 2002;137:1-10. doi: 10.7326/0003-4819-137-1200207020-00006.

14. Ceriotti F, Henny J, Queralto J, Ziyu S, Ozarda Y, Chen $\mathrm{B}$, et al. Common reference intervals for aspartate aminotransferase (AST), alanine aminotransferase (ALT) and gamma-glutamyl transferase (GGT) in serum: results from an IFCC multicenter study. Clin Chem Lab Med 2010;48:1593-601. doi: 10.1515/CCLM.2010.315.

15. Beynon R, Leeflang MM, McDonald S, Eisinga A, Mitchell RL, Whiting P, et al. Search strategies to identify diagnostic accuracy studies in MEDLINE and EMBASE. Cochrane Database Syst Rev 2013;9:Mr000022. doi: 10.1002/14651858.MR000022.pub3.

16. Volpato ES, Betini M, El Dib R. Testing search strategies for systematic reviews in the Medline literature database through PubMed. J Eval Clin Pract 2014;20:117-20. doi:10.1111/jep.12094.

17. Moher D, Liberati A, Tetzlaff J, Altman DG, The PG. Preferred Reporting Items for Systematic Reviews and Meta-Analyses: The PRISMA Statement. BMJ 2009;339:b2535. doi: 10.1136/bmj.b2535.

18. Al-hamoudi W, Ali S, Hegab B, Elsiesy H, Hashim A, Al-Sofayan M, et al. Revising the upper limit of normal for levels of serum alanine aminotransferase in a Middle Eastern population with normal liver histology. Dig Dis Sci 2013;58:2369-75. doi: 10.1007/s10620-013-2659-0.

19. Brinkmann T, Dreier J, Diekmann J, Gotting C, Klauke R, Schumann G, et al. Alanine aminotransferase cut-off values for blood donor screening using the new International Federation of Clinical Chemistry reference method at 37 degrees C. Vox Sang 2003;85:159-64. doi:10.1046/j.1423-0410.2003.00347.x 
20. Choudhary NS, Saraf N, Saigal S, Gautam D, Lipi L, Soin AS. Estimation of normal values of serum transaminases based on liver histology in healthy Asian Indians. $J$ Gastroenterol Hepatol 2015;30:763-6. doi:10.1111/jgh.12836.

21. Dorizzi RM, Tagliaro F, Capuzzo E, Disperati A. Serum alanine transaminase (ALT) reference range in Italy. J Clin Pathol 1991;44:790-1. doi: 10.1136/jcp.44.9.790-b.

22. George J, Denney-Wilson E, Okely AD, Hardy LL, Aitken R. The population distributions, upper normal limits and correlations between liver tests among Australian adolescents. J Paediatr Child Health 2008;44:579-85. doi:10.1111/j.1440-1754.2008.01386.x

23. Goldie DJ, McConnell AA. Serum alanine transaminase (ALT) reference ranges estimated from blood donors. $J$ Clin Pathol 1990;43:929-31. doi:10.1136/jcp.43.11.929.

24. Grunenberg R, Banik N, Kruger J. Alanine aminotransferase (ALAT, GPT): a reevaluation of exclusion limits for blood donors. Infusionsther Transfusions med 1995;22:145-51.

25. Helmersson-Karlqvist J, Ridefelt P, Lind L, Larsson A. Reference values for 34 frequently used laboratory tests in 80-year-old men and women. Maturitas 2016;92:97-101. doi: 10.1016/j.maturitas.2016.07.015.

26. Hilsted L, Rustad P, Aksglaede L, Sorensen K, Juul A. Recommended Nordic paediatric reference intervals for 21 common biochemical properties. Scand J Clin Lab Invest 2013;73:1-9. doi: 10.3109/00365513.2012.721519.

27. Ibrahim K, Yousufi MA, Hasnain SN, Zuberi SJ. Serum aspartate amino transferase and alanine amino transferase levels in apparently healthy population in Karachi. $J$ Pak Med Assoc 1988:38:325-7.

28. Jamali R, Pourshams A, Amini S, Deyhim MR, Rezvan H, Malekzadeh R. The upper normal limit of serum alanine aminotransferase in Golestan Province, northeast Iran. Arch Iran Med 2008;11:602-7.

29. Kabir A, Pourshams A, Khoshnia M, Malekzadeh F. Normal limit for serum alanine aminotransferase level and distribution of metabolic factors in old population of Kalaleh, Iran. Hepat Mon 2013;13:e10640. doi: 10.5812/hepatmon.10640.

30. Khan FA, Dilawar M, Khan DA. Reference values of common blood chemistry analytes in healthy population of Rawalpindi-Islamabad area. J Pak Med Assoc 1997;47:156-9.

31. Khedmat H, Fallahian F, Abolghasemi H, Hajibeigi B, Attarchi Z, Alaeddini F, et al. Serum gamma-glutamyltransferase, alanine aminotransferase, and aspartate aminotransferase activity in Iranian healthy blood donor men. World J Gastroenterol 2007;13:889-94. doi: 10.3748/wjg.v13.i6.889.

32. Kumar S, Amarapurkar A, Amarapurkar D. Serum aminotransferase levels in healthy population from western India. Indian J Med Res 2013;138:894-9.
33. Lai DS, Chen SC, Chang YH, Chen CY, Lin JB, Lin YJ, et al. Pediatric reference intervals for several biochemical analytes in school children in Central Taiwan. J Formos Med Assoc 2009;108:957-63. doi: 10.1016/S0929-6646(10)60009-5.

34. Laouina A, Abouyoub A, Soulaymani A, Alami R. Alanine transaminase level in a healthy population in Morocco. Pak J Biol Sci 2012;15:238-43. doi: 10.3923/ pjbs.2012.238.243.

35. Lee JK, Shim JH, Lee HC, Lee SH, Kim KM, Lim YS, et al. Estimation of the healthy upper limits for serum alanine aminotransferase in Asian populations with normal liver histology. Hepatology 2010;51:1577-83. doi: 10.1002/hep.23505.

36. Leino A, Impivaara O, Irjala K, Maki J, Peltola O, Jarvisalo J. Health-based reference intervals for ALAT, ASAT and GT in serum, measured according to the recommendations of the European Committee for Clinical Laboratory Standards (ECCLS). Scand J Clin Lab Invest 1995;55:243-50. doi: 10.3109/00365519509089619.

37. Lozano M, Cid J, Bedini JL, Mazzara R, Gimenez N, Mas $\mathrm{E}$, et al. Study of serum alanine-aminotransferase levels in blood donors in Spain. Haematologica 1998;83:237-9.

38. Mijovic V, Contreras M, Barbara J. Serum alanine aminotransferase (ALT) and gamma-glutamyltransferase (gammaGT) activities in north London blood donors. J Clin Pathol 1987;40:1340-4. doi: 10.1136/jcp.40.11.1340.

39. Mohamadnejad M, Pourshams A, Malekzadeh R, Mohamadkhani A, Rajabiani A, Asgari AA, et al. Healthy ranges of serum alanine aminotransferase levels in Iranian blood donors. World J Gastroenterol 2003;9:2322-4. doi: 10.3748/wjg.v9.i10.2322.

40. Park HN, Sinn DH, Gwak GY, Kim JE, Rhee SY, Eo SJ, et al. Upper normal threshold of serum alanine aminotransferase in identifying individuals at risk for chronic liver disease. Liver Int 2012;32:937-44. doi: 10.1111/j.14783231.2011.02749.x

41. Park SH, Heo NY, Kim CH, Suk KT, Kim DJ, Lee HY. Upper reference limits for aminotransferase activities and the prevalence of elevated aminotransferase activities in a Korean population. J Clin Gastroenterol 2013;47:76-82. doi: 10.1097/MCG.0b013e31825752a4.

42. Poustchi H, George J, Esmaili S, Esna-Ashari F, Ardalan G, Sepanlou SG, et al. Gender Differences in Healthy Ranges for Serum Alanine Aminotransferase Levels in Adolescence. PLoS One 2011;6:e21178. doi: 10.1371/ journal.pone.0021178.

43. Rodoo P, Ridefelt P, Aldrimer M, Niklasson F, Gustafsson J, Hellberg D. Population-based pediatric reference intervals for HbAlc, bilirubin, albumin, CRP, myoglobin and serum enzymes. Scand J Clin Lab Invest 2013;73:361-7. doi: 10.3109/00365513.2013.783931.

44. Ruhl CE, Everhart JE. Trunk fat is associated with increased 
serum levels of alanine aminotransferase in the United States. Gastroenterology 2010;138:1346-56.e3 doi: 10.1053/j.gastro.2009.12.053.

45. Rustad P, Felding P, Franzson L, Kairisto V, Lahti A, Martensson A, et al. The Nordic Reference Interval Project 2000: recommended reference intervals for 25 common biochemical properties. Scand J Clin Lab Invest 2004;64:271-84. doi: 10.1080/00365510410006324.

46. Sohn W, Jun DW, Kwak MJ, Park Q, Lee KN, Lee HL, et al. Upper limit of normal serum alanine and aspartate aminotransferase levels in Korea. $J$ Gastroenterol Hepatol 2013;28:522-9. doi: 10.1111/j.1440-1746.2012.07143.x

47. Van der Poorten D, Kenny DT, Butler T, George J. Liver disease in adolescents: A cohort study of high-risk individuals. Hepatology 2007;46:1750-8. doi:10.1002/hep.21918.

48. Vespasiani-Gentilucci U, Gallo P, Piccinocchi G, Piccinocchi R, Schena E, Galati G, et al. Determinants of alanine aminotransferase levels in a large population from Southern Italy: Relationship between alanine aminotransferase and age. Dig Liver Dis 2014;46:909-15. doi: 10.1016/j.dld.2014.05.021.

49. Wu WC, Wu CY, Wang YJ, Hung HH, Yang HI, Kao WY, et al. Updated thresholds for serum alanine aminotransferase level in a large-scale population study composed of 34 346 subjects. Aliment Pharmacol Ther 2012;36:560-8. doi: 10.1111/j.1365-2036.2012.05224.x

50. Zhang GM, Xia YJ, Guo XX, Zhu BL, Zhang GM, Ma $\mathrm{XB}$, et al. Reference intervals for total bilirubin, ALT, AST and creatinine in healthy Chinese elderly. Med Sci Monit 2014;20:1778-82. doi: 10.12659/MSM.892148.

51. Zhang P, Wang CY, Li YX, Pan Y, Niu JQ, He SM. Determination of the upper cut-off values of serum alanine aminotransferase and aspartate aminotransferase in Chinese. World $J$ Gastroenterol 2015;21:2419-24. doi: 10.3748/wjg.v21.i8.2419.

52. Zheng M-H, Shi K-Q, Fan Y-C, Liu W-Y, Lin X-F, Li L-F, et al. Upper Limits of Normal for Serum Alanine Aminotransferase Levels in Chinese Han Population. PLoS One 2012;7:e43736. doi: 10.1371/journal.pone.0043736.

53. Kang HS, Um SH, Seo YS, An H, Lee KG, Hyun JJ, et al. Healthy range for serum ALT and the clinical significance of "unhealthy" normal ALT levels in the Korean population. J Gastroenterol Hepatol 2011;26:292-9. doi:10.1111/ j.1440-1746.2010.06481.x

54. Abebe M, Melku M, Enawgaw B, Birhan W, Deressa T, Terefe B, et al. Reference intervals of routine clinical chemistry parameters among apparently healthy young adults in Amhara National Regional State, Ethiopia. PLoS One 2018;13:e201782. doi:10.1371/journal.pone.0201782.

55. Achila OO, Semere P, Andemichael D, Gherezgihier $\mathrm{H}$, Mehari S, Amanuel A, et al. Biochemistry reference intervals for healthy elderly population in Asmara, Eritrea.
BMC Res Notes 2017;10:748. doi: 10.1186/s13104-017-3087-6.

56. Bussler S, Vogel M, Pietzner D, Harms K, Buzek T, Penke $M$, et al. New pediatric percentiles of liver enzyme serum levels (alanine aminotransferase, aspartate aminotransferase, gamma-glutamyltransferase): Effects of age, sex, body mass index, and pubertal stage. Hepatology 2018;68:131930. doi: 10.1002/hep. 29542 .

57. Balachiranjeevi C H , Bhaskar Naik S , Abhilash Kumar V , Harika G , Mahadev Swamy H K , Hajira Sk , et al. Marker-assisted pyramiding of two major, broadspectrum bacterial blight resistance genes, Xa21 and Xa33 into an elite maintainer line of rice, DRR17B. PLoS One 2018;13:e0201271. doi: 10.1371/journal.pone.0201271.

58. Li Y, Mussa AE, Tang A, Xiang Z, Mo X. Establishing reference intervals for ALT, AST, UR, Cr, and UA in apparently healthy Chinese adolescents. Clin Biochem 2018;53:72-6. doi:10.1016/j.clinbiochem.2018.01.019.

59. Li X, Wang D, Yang C, Zhou Q, Zhuoga SL, Wang LQ, et al. Establishment of age- and gender-specific pediatric reference intervals for liver function tests in healthy Han children. World J Pediatr 2018;14:151-9. doi: 10.1007/ s12519-018-0126-x

60. Dosoo DK, Asante KP, Kayan K, Adu-Gyasi D, Osei-Kwakye $\mathrm{K}$, Mahama E, et al. Biochemical and hematologic parameters for children in the middle belt of Ghana. Am J Trop Med Hyg 2014;90:767-73. doi: 10.4269/ajtmh.13-0098.

61. Mofrad P, Contos MJ, Haque M, Sargeant C, Fisher RA, Luketic VA, et al. Clinical and histologic spectrum of nonalcoholic fatty liver disease associated with normal ALT values. Hepatology 2003;37:1286-92. doi: 10.1053/jhep.2003.50229.

62. Piton A, Poynard T, Imbert-Bismut F, Khalil L, Delattre J, Pelissier E, et al. Factors associated with serum alanine transaminase activity in healthy subjects: consequences for the definition of normal values, for selection of blood donors, and for patients with chronic hepatitis C. MULTIVIRC Group. Hepatology 1998;27:1213-9. doi: 10.1002/hep.510270505.

63. Schwimmer JB, McGreal N, Deutsch R, Finegold MJ, Lavine JE. Influence of gender, race, and ethnicity on suspected fatty liver in obese adolescents. Pediatrics 2005;115:561-5. doi:10.1542/peds.2004-1832.

64. Fraser A, Longnecker MP, Lawlor DA. Prevalence of elevated alanine aminotransferase among US adolescents and associated factors: NHANES 1999-2004. Gastroenterology 2007;133:1814-20. doi: 10.1053/j.gastro.2007.08.077.

65. Kunde SS, Lazenby AJ, Clements RH, Abrams GA. Spectrum of NAFLD and diagnostic implications of the proposed new normal range for serum ALT in obese women. Hepatology 2005;42:650-6. doi: 10.1002/hep.20818.

66. Vento S, Nobili V. Aminotransferases as predictors of mortality. Lancet 2008;371:1822-3. doi: 10.1016/S01406736(08)60778-3. 